Artigo Original

\title{
Validação de um protocolo de avaliação do tono muscular e atividades funcionais para crianças com paralisia cerebral
}

\author{
Durigon, O.F. S. ; Sá, C.S.S. ${ }^{2}$ Sitta, L.V. ${ }^{3}$
}

\begin{abstract}
RESUMO
Em disfunções neurológicas, tem sido um desafio medir as mínimas mudanças nas atividades funcionais de crianças submetidas a tratamento fisioterapêutico. Em nosso ponto de vista isto se deve ao fato de que as escalas desenvolvidas até então procuram avaliar funções ou habilidades e não o desempenho motor implícito na função.

Considerando que este estudo objetivou testar a aplicabilidade de um protocolo de avaliação que medisse o tono muscular e a funcionalidade em crianças com Paralisia Cerebral. Foi usada a escala do tono muscular previamente testada em adultos e a escala da funcionalidade previamente desenvolvida para crianças com Síndrome de Rett que foi adaptada para crianças com Paralisia Cerebral. Quarenta e quatro crianças receberam fisisioterapia e foram avaliadas. A confiabilidade do protocolo em relação às escalas usadas previamente foram analisadas através da sua porcentagem de concordância. Como nós obtivemos $100 \%$ de concordânica com os 5 examinadores no teste de Johnstone e principalmente porque esta ferramena também tem validade de construto e se mostrou sensível a mudanças ocorridas na terapia, este protocolo pode ser considerado válido e confiável na avaliação motora e desempenho funcional das crianças com Paralisia Cerebral.
\end{abstract}

Unitermos: Tono muscular, Atividades funcionais, Avaliação, Paralisia cerebral, Criança

\section{SUMMARY}

In neurological disfunctions has been difficult to measure the minimal changes in the functional activities of children submitted to physiotherapic treatment. In our point of view, this is happening because the scales developed so far pretended to evaluate functions or habilities but the implicit motor performance in that function.

Considering that, this study aimed to test the applicability of a evaluation protocol that measures the muscular tone and functionality in Cerebral Palsy children. It was used a muscular tone scale previously tested in adults and a functional scale previously developed for Rett Syndrome children which was adapted for Cerebral Palsy children. Forty-four Cerebral Palsy children receiving physical therapy were evaluated. The reliability of the protocol in relation to previously used scales degrees were analyzed through their percentage of concordance. As we got $100 \%$ of concordance with five examinators in the Johnstone test and principally because this tool has its construsto validity and behaved sensitively to the occurred changes in therapy, this protocol can be considered valid and reliable for evaluating motor and functional performance in Cerebral Palsy children.

Keywords: Muscle tone, Functional activities, Assessment, Cerebral palsy, Child.

\footnotetext{
Local: Laboratório de Disfunções Neurológicas Infantis - CDP - FMUSP.

1 - Profa. Doutora do Curso de Fisioterapia do Departamento de Fisioterpaia, Fonoaudiologia e Terapia Ocupacional da Faculdade de Medicina da USP 2 - Profa. do Curso de Fisioterapia do IMES, doutoranda em Neurociências e comportamento -USP, Profa. convidado do Curso de Avançado em Fisioterapia Neurológica da USP

3 - Fisioterapeutas, doutorandas em Neurociências e Comportamento pela Universidade de São Paulo
}

Correspondência: Departamento de Fisioterapia, Fonoaudiologia, Terapia Ocupacional

Rua: Cipotânea 51, Cidade Universitária "Armando Salles de Oliveira" - CEP: 05360-000 - São Paulo - SP - Brasil - e-mail: ofsdurigon@uol.com.br 


\section{INTRODUÇÃO}

É de senso comum que crianças com Paralisia Cerebral (PC) beneficiam-se de programas de tratamento fisioterapêutico, especialmente quando iniciados precocemente na vida (1). Para tanto, é necessário diagnosticar essas crianças, identificar suas necessidades e planejar um tratamento que estimule seu desenvolvimento ${ }^{(2)}$.

Para que essas etapas do programa de tratamento sejam cumpridas com eficiência, é necessária a existência de métodos adequados de avaliação. A literatura relata diversos métodos de avaliação específicos ou não para crian-

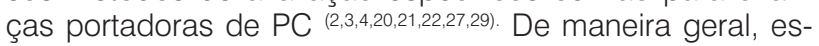
ses métodos podem ser classificados em dois grupos: 1 medidas discriminativas, ou seja, métodos que buscam definir ou não a presença de determinadas características ou funções e 2 - medidas avaliativas, métodos usados para medir a magnitude das mudanças ocorridas no desenvolvimento. Ao utilizar um método de avaliação, é importante estabelecer qual o seu propósito.

Existem diversos métodos de avaliação do desenvolvimento motor descritos atualmente na literatura e utilizados com freqüência, entre eles Denver Developmental Screening Tests (DDST), o Bayley Motor Scale (BMS), o Movement Assessment of Infants (MAI), o Infant Motor Screen (IMS), o Battelle Developmental Inventory Screening Test (BDIST), o Peabody Development Motor Scale (PDMS). Estas escalas, em sua maioria privilegiam a identificação precoce de crianças com sinais de anormalidades ou até mesmo o diagnóstico de PC. Com esse objetivo principal estão o Denver ${ }^{(13)}$., o $\mathrm{BMS}^{(22)}$., o MAI(22). , o IMS ${ }^{(27)}$ e o BDIST ${ }^{(16)}$., cada uma com suas peculiaridades. Somente o PDMS ${ }^{(28)}$. propõe-se a avaliação da evolução do desenvolvimento em resposta à terapia, mas não cumpre completamente o proposto, pois não responde às alterações no desenvolvimento ocorridas à curto prazo em crianças com um maior comprometimento.

Além dessas escalas já validadas na literatura, outras escalas estão sendo construídas atualmente. A Alberta Infant Motor Scale (AIMS) ${ }^{(29)}$ enfoca a seqüência do desenvolvimento do controle postural através de 58 itens, propondo: 1 - identificar atrasos de desenvolvimento; 2 - prover informações a médicos e pais a cerca das atividades motoras da criança; 3 - mensurar o desempenho motor antes, durante e após o tratamento; 4 - medir pequenas mudanças na performance não detectáveis por métodos tradicionais e 5 - atuar como um instrumento de pesquisa apropriado para avaliar a eficácia de programas de reabilitação ${ }^{(29)}$

Segundo os autores da AIMS ${ }^{(29)}$, tanto o desenvolvimento motor quanto suas alterações seriam melhor avaliadas através da análise dos componentes usados para alcançar marcos do desenvolvimento. Assim, a AIMS(29) também propõe-se a mensurar a evolução das crianças submetidas à tratamento fisioterapêutico.

O Gross Motor Function Measure (GMFM) ${ }^{(6,20,32,35)}$ também foi desenvolvido para ser uma medida avaliativa e foi validado para detectar mudanças na motricidade grossa de crianças com PC para fins clínicos e de pesquisa. Consiste de 85 itens divididos em 8 grupos (supino, prono, quatro apoios, sedestação, ajoelhado, bipedestação, marcha e subida/descida). Essa escala foi desenvolvida para avaliar a função quantitativamente, sem considerar seu aspecto qualitativo, visto a complexidade da qualidade do movimento ${ }^{(32)}$.
Segundo os autores do GMFM $^{(6,20,32)}$, com essa deliberada exclusão dos componentes qualitativos, diversas mudanças não são refletidas no escore total. Apesar disso, a correlação entre os escores de mudanças no desenvolvimento refletidas por esse instrumento e as mudanças relatadas por pais e terapeutas, suportam a hipótese de que esse método é sensível a mudanças ${ }^{(32)}$.

A fim de avaliar o aspecto qualitativo, os autores da GMFM estão desenvolvendo o GMPM (Gross Motor Performance Measure) para ser usado em conjunto com o GMFM. O objetivo do GMPM é ser uma medida genérica, sem vínculos com teorias de controle motor, que possa documentar o grau de mudança no desempenho motor de crianças submetidas à intervenção. As críticas a essa escala são a rejeição da estruturação teórica, pois a terminologia utilizada não tem sentido fora de um contexto téorico e a subjetividade do escore. (4)

Diante dessa análise de métodos de avaliação descritos na literatura, nota-se a importância em definir detalhadamente os objetivos propostos para uma escala. A escoIha correta de um método de avaliação inclui primeiramente determinar o objetivo dessa avaliação, para então escoIher um método considerado cientificamente válido para o objetivo proposto.

Além da função, outro aspecto que deveria ser avaliado nas crianças com PC é o tono muscular. Das escalas discutidas acima, apenas o MAI e o IMS avaliam o tono muscular, por terem objetivos discriminativos. Nas medidas de avaliação das crianças com PC, é evidente a ênfase na avaliação da funcionalidade em detrimento da avaliação do tono muscular.

O número restrito de métodos de avaliação detalhada do tono muscular de adultos ou crianças dificulta a verificação da veracidade dessas afirmações. A literatura relata que existem alguns métodos qualitativos e quantitativos de mensuração da hipertonia ${ }^{(8)}$. Apesar dos aspectos positivos de cada um deles, pode-se considerar que nenhum deles reflete exatamente o que ocorre no SNC e daí a fragilidade dos mesmos.

A partir desses instrumentos de avaliação do tono muscular e da funcionalidade descritos acima, é evidente a ausência de um método de avaliação do desempenho motor que avalie as mudanças ocorridas no tono muscular e nas atividades funcionais de crianças com PC.

Da ausência de uma medida avaliativa decorre a dificuldade em mensurar a resposta da criança à Fisioterapia. Sem procedimentos científicos e objetivos para acompanhar quantitativamente a evolução de uma criança. Diante dessa necessidade, procuramos desenvolver um método de avaliação do tono muscular e das atividades funcionais que tivesse a capacidade de expressar a evolução das crianças com PC sendo submetidas à Fisioterapia.

A fim de objetivar a avaliação do grau de hipertonia, seria necessário criar uma escala que possibilitasse uma graduação objetiva. Essa escala foi desenvolvida previamente em adultos e reflete a atividade do reflexo miotático, o que em última análise reflete a atividade neural resultante do desequilíbrio que gerou a alteração de tono muscular(8). 
Segundo os autores dessa escala, esse sistema de avaliação e classificação foi baseado em fundamentos neurofisiológicos associados à observação clínica. Para sua elaboração levou-se em consideração ${ }^{(8)}$ : a capacidade de adaptação do fuso muscular ao movimento; a intensidade da reação ao alongamento; a variação da manifestação da hipertonia com a variação da velocidade do movimento.

A sua aplicação em crianças, além de fornecer uma medida quantitativa, possibilitaria a avaliação da evolução do tono muscular das crianças com PC submetidas à Fisioterapia. Essa mensuração mais objetiva do tono muscular responderia entre outras coisas à questão se ocorre ou não modulação do tono muscular acompanhando as aquisições motoras de crianças hipertônicas. Seria então possível inferir se a aparente diminuição da hipertonia resulta de adaptações no controle tônico ou apenas de uma melhora do controle do movimento sobre um tono muscular alterado. Este último dado permitiria verificar a eficiência das diferentes concepções terapêuticas nas diferentes fases da terapia.

A fim de objetivar a avaliação do grau de funcionalidade, foi necessário criar uma escala que possibilitasse uma avaliação quantitativa e qualitativa das atividades funcionais. Essa escala foi baseada no protocolo de avaliação descrito inicialmente para crianças com outras patologias neurológicas $^{(10)}$ e envolveu atividades estáticas e dinâmicas comumente utilizadas pelos fisioterapeutas na avaliação e tratamento das crianças com PC.

Esse protocolo de avaliação das atividades funcionais enfatizou a independência e o controle postural envolvendo o alinhamento, estabilidade e movimento nas posturas das crianças com PC. Em virtude disso, ao construir esse protocolo, levou-se em consideração os mecanismos neurofisiológicos de ajuste postural e fases de aquisições, que acham-se muitíssimo bem definidas por Shumway-Cook e Woollacott(32).

As atividades funcionais avaliadas nesse protocolo representam a seqüência do desenvolvimento neuromotor. A capacidade de realização dessas atividades envolve a utilização integrada de diversos ajustes posturais, constituindo a base tônico-postural necessária para a realização das atividades motoras em geral.

Do que foi exposto até aqui, fica claro que os meios de atuação do fisioterapeuta nas disfunções neurológicas dependem do desenvolvimento dos sistemas da avaliação. 0 Curso de Fisioterapia da Universidade de São Paulo vem trabalhando no desenvolvimento desses sistemas. Já foram desenvolvidos um protocolo de avaliação do tono muscular em adultos ${ }^{(8)}$ e um protocolo de avaliação da funcionalidade de crianças com outras patologias neurológicas ${ }^{(10)}$, que foi adaptado em um estudo piloto para ser utilizado em crianças com PC ${ }^{(9)}$. Este estudo vincula-se a esse projeto e visa a construção de um método de avaliação "semi-quantitativo", sensível a evolução do desempenho motor e funcional de crianças sendo submetidas a Fisioterapia. Para tanto, o objetivo desse estudo é determinar a aplicabilidade dos protocolos de avaliação do tono muscular e das atividades funcionais em crianças com diagnóstico de PC (11)

\section{MÉTODO}

Participaram desse estudo todas as crianças $(n=44) \mathrm{com}$ diagnóstico de Paralisia Cerebral em tratamento fisioterapêutico no Ambulatório de Fisioterapia em Neurologia do Centro de Docência e Pesquisa em Fisioterapia da Faculdade de Medicina da Universidade de São Paulo (CDP - FMUSP). Este procedimento faz parte da rotina de avaliação de crianças com este tipo de disfunção e foram autorizados a participar deste estudo pelos pais ou responsáveis. A idade das crianças variou de 5 a 148 meses (média de 42,9 meses). As crianças foram avaliadas em uma sala ampla e bem iluminada, no Laboratório de Disfunções Neurológicas Infantis do CDP - FMUSP.

Cinco terapeutas aplicaram os procedimentos de cada protocolo com a finalidade de estabelecer o grau de concordância através da fórmula $(I=A / A+B+C+D \times 100)$, ou seja, índice de Jonhstone.

Foram utilizados os protocolos de avaliação do tono muscular (Durigon e Piemonte, 1993) e das atividades funcionais (Durigon, Sá e Sita, 1996, 1999), colchonete, brinquedos diversos e um banco de madeira adequado à altura da criança apenas para as atividades funcionais.

As crianças foram avaliadas individualmente levando-se em conta duas variáveis: tono muscular e funcionalidade.

\section{- Avaliação do Tono Muscular}

O tono muscular foi classificado através da aplicação de uma escala ordinal (Tabela1) que leva em conta a "reação ao alongamento". Essa escala foi desenvolvida em adultos ${ }^{(8)}$, testada em crianças com PC em um estudo piloto e pretende ser validada neste estudo.

A criança orientada em relação ao procedimento realizado em sala desprovida de estímulos visuais ou distratores, e em seguida era posicionada alinhada em DD, mantendo seu olhar orientado para cima, não se permitindo sua mobilidade durante o exame. $\mathrm{O}$ fisioterapeuta procedia à avaliação dos grupos musculares utilizando a mobilização passiva segmentar unilateral, não utilizando comandos verbais durante cada passo do procedimento. Em um primeiro movimento, foi realizada a mobilização súbita da articulação e um segundo movimento utilizando a mobilização lenta era empregado quando se necessitasse.

\section{- Avaliação das Atividades Funcionais}

O protocolo de avaliação das atividades funcionais foi baseado no protocolo de avaliação da funcionalidade descrito inicialmente para outras patologias neurológicas ${ }^{(10)}$. Em virtude da limitação funcional dos pacientes com outras patologias, essa escala necessitou ser previamente ampliada para utilização nas crianças com diagnóstico de PC. Para tanto, realizamos um estudo prévio ${ }^{(9)}$ no qual avaliamos nove crianças de acordo com aspectos quantitativos e qualitativos das atividades estáticas e dinâmicas não propostas no protocolo inicial e propusemos uma escala mais abrangente e adequada para a amostra de crianças com diagnóstico de PC (Tabelas 2 e 3). Nesta fase, realizamos a avaliação de mais 21 sujeitos, de modo a validar ou corrigir os graus propostos no estudo piloto. 


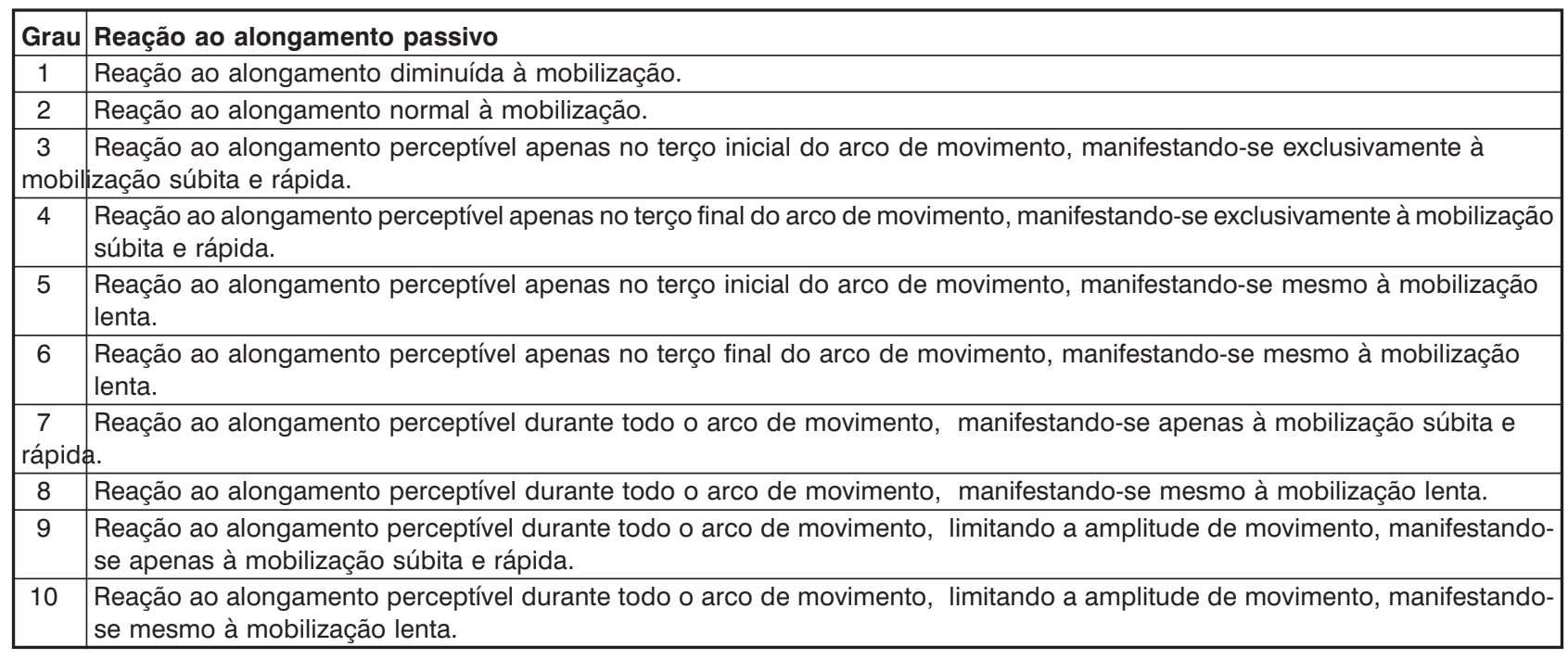

Tabela 1 - Escala Ordinal de Avaliação do Tono Muscular desenvolvida em adultos.

\begin{tabular}{|c|l|}
\hline Grau & Adoção e manutenção da postura \\
\hline 0 & não realiza a atividade funcional \\
\hline 1 & adota a postura com auxílio, mantém com apoio e sem alinhamento \\
\hline 2 & adota a postura com auxílio, mantém com apoio e com alinhamento \\
\hline 3 & adota a postura com auxílio, mantém sem apoio e sem alinhamento \\
\hline 4 & adota a postura com auxílio, mantém sem apoio e com alinhamento \\
\hline 5 & adota a postura sem auxílio, mantém com apoio e sem alinhamento \\
\hline 6 & adota a postura sem auxílio, mantém com apoio e com alinhamento \\
\hline 7 & adota a postura sem auxílio, mantém sem apoio e sem alinhamento \\
\hline 8 & adota a postura sem auxílio, mantém sem apoio e com alinhamento \\
\hline 9 & adota a postura sem auxílio, mantém sem apoio e realiza atividades na postura mantendo o alinhamento \\
\hline
\end{tabular}

Tabela 2 - Escala de Avaliação das Atividades Funcionais Estáticas (sedestação, quadrupedia, ajoelhado, semi-ajoelhado e bipedestação) desenvolvida para a amostra de crianças com Paralisia Cerebral

\begin{tabular}{|c|l|}
\hline Grau & Realização da atividade funcional \\
\hline 0 & Não realiza a atividade funcional \\
\hline 1 & Realiza a atividade em "padrão anormal" \\
\hline 2 & Realiza a atividade em padrão normal e coordenado, \\
\hline & porém com alterações qualitativas \\
\hline
\end{tabular}

(engatinhar e marcha) desenvolvida para a amostra de crianças com Paralisia Cerebral

Nas atividades estáticas (sedestação, quadrupedia, ajoelhado e semiajoelhado, bipedestação) foram utilizados como parâmetros quantitativos a capacidade de adoção e manutenção da postura e como parâmetros qualitativos a independência e o alinhamento postural. Para tanto, utilizouse uma graduação objetiva do aspecto qualitativo, limitando-se à classificação da presença ou ausência de independência e alinhamento, evitando itens subjetivos que comprometeriam a validade da graduação.

Nas atividades dinâmicas (engatinhar e marcha), foi considerado o parâmetro quantitativo de capacidade de realização da atividade e como parâmetro qualitativo a presença ou ausência de normalidade no padrão de realização.

Cada criança foi orientada e/ou incentivada a adotar todas as posturas, na seqüência do desenvolvimento motor, utilizando-se ordens claras, bem como espelhos e brinquedos como incentivo.

\section{ANÁLISE DOS DADOS}

Os resultados individuais foram classificados a partir dos parâmetros das escalas utilizadas, verificando a sua aplicabilidade ou não nos pacientes da nossa amostra, uma vez que ambas foram construídas para outro tipo de amostra.

Este procedimento poderia confirmar ou não a fidedignidade dos parâmetros servindo para validá-los, ampliá-los ou corrigí-los.

Inicialmente, foram analisados os dados referentes ao tono muscular, com relação a porcentagem de grupos musculares avaliados em cada grau descrito na Escala de Avaliação do Tono Muscular. A partir disso, calculou-se a porcentagem de concordância com os graus atribuídos para a escala, verificando a validade da escala para nossa amostra.

Posteriormente analisamos os dados referentes às atividades funcionais de todos os sujeitos avaliados, incluindo 
os sujeitos avaliados no estudo piloto ${ }^{(9)}$. Os sujeitos foram divididos em grupos segundo os resultados obtidos com a avaliação dos parâmetros quantitativos e qualitativos propostos. A seguir, foi calculada a somatória das atividades funcionais estáticas de todos os sujeitos, buscando seu agrupamento segundo graus de funcionalidade. O mesmo foi feito com relação às atividades funcionais dinâmicas. Finalmente, calculou-se a porcentagem de concordância entre os graus atribuídos a essa amostra e os graus propostos no estudo piloto ${ }^{(9)}$, a fim de verificar a validade da Escala de Atividades Funcionais para crianças com PC.

\section{RESULTADOS}

O Quadro 1 demostra os resultados obtidos pelos 5 avaliadores para o procedimento de avaliação do tono muscular para os membros superiores direito e esquerdo respectivamente. Da mesma forma o Quadro 2 demonstra os resultados obtidos pelos 5 avaliadores para os membros inferiores esquerdo e direito. A partir da visualização dos quadros observamos $100 \%$ de concordância entre os avaliadores.

Os Quadros 3 e 4 registram os resultados obtidos pelos 5 avaliadores durante as avaliações funcionais estáticas e dinâmicas respectivamente.

\section{A. Dados referentes à Avaliação de Tono Muscular}

Foram analisados 24 grupos musculares, bilateralmente, em cada um dos 44 sujeitos, totalizando 2112 grupos

\begin{tabular}{|l|c|c|c|c|c|}
\hline Grupos musculares & Ex.1 & Ex.2 & Ex.3 & Ex.4 & Ex.5 \\
\hline flexores de ombro & 2 & 2 & 2 & 2 & 2 \\
\hline extensores de ombro & 2 & 2 & 2 & 2 & 2 \\
\hline adutores de ombro & 3 & 3 & 3 & 3 & 3 \\
\hline abdutores de ombro & 2 & 2 & 2 & 2 & 2 \\
\hline rotadores medial de ombro & 3 & 3 & 3 & 3 & 3 \\
\hline rotadores lateral de ombro & 2 & 2 & 2 & 2 & 2 \\
\hline flexores de cotovelo & 3 & 3 & 3 & 3 & 3 \\
\hline extensores de cotovelo & 2 & 2 & 2 & 2 & 2 \\
\hline pronadores & 3 & 3 & 3 & 3 & 3 \\
\hline supinadores & 2 & 2 & 2 & 2 & 2 \\
\hline flexores de punho & 3 & 3 & 3 & 3 & 3 \\
\hline extensores de punho & 2 & 2 & 2 & 2 & 2 \\
\hline flexores de dedos & 3 & 3 & 3 & 3 & 3 \\
\hline extensores de dedo & 2 & 2 & 2 & 2 & 2 \\
\hline Ex. = Examinador & & & \\
\hline
\end{tabular}

Quadro 1 - Representação dos resultados da avaliação do tono muscular do membro superior esquerdo realizada pelos 5 examinadores.

\begin{tabular}{|l|c|c|c|c|c|}
\hline Atividades & Ex.1 & Ex.2 & Ex.3 & Ex.4 & Ex.5 \\
\hline Sedestação & 8 & 8 & 8 & 8 & 8 \\
\hline Quadrupedia & 6 & 6 & 6 & 6 & 6 \\
\hline Ajolhado & 5 & 5 & 5 & 5 & 5 \\
\hline Semiajoelhado & 1 & 1 & 1 & 1 & 1 \\
\hline Bipedestação & 7 & 7 & 7 & 7 & 7 \\
\hline Ex. = Examinador & \multicolumn{5}{|l}{} \\
\hline
\end{tabular}

Quadro 3 - Representação dos dados referentes a avaliação das atividades funcionais estáticas realizada pelos cinco examinadores. musculares avaliados e classificados segundo a Escala de Avaliação de Tono Muscular, concordando estes em 100\% na avaliação feito por 5 examinadores, sendo estatisticamente comprovada pelo índice de Jonhston $(I=A / A+B+C+D$ $\mathrm{x} 100)$

A partir dos resultados, calculou-se a porcentagem de concordância com os graus atribuídos para a Escala de Avaliação do Tono Muscular desenvolvida em adultos, sendo a mesma de $100 \%$, visto que todos os grupos musculares puderam ser classificados nos graus descritos pela escala.

Assim, baseado na porcentagem de concordância, consideramos que a Escala de Avaliação do Tono Muscular desenvolvida em adultos é aplicável em crianças com PC.

\section{B. Dados referentes à Avaliação das Atividades Funcionais}

A porcentagem de concordância entre os graus atribuídos a essa amostra e os graus propostos no estudo piloto (9) foi de $100 \%$, visto que toda a nossa amostra enquadrou-se nos graus propostos pela Escala de Atividades Funcionais Estáticas, não havendo necessidade de modificação da escala proposta pelo estudo piloto ${ }^{(9)}$. Assim, consideramos a Escala de Atividades Funcionais aplicável em crianças com PC.

Esta escala também concordou em 100\% na avaliação feita por 5 examinadores, sendo estatisticamente comprovada pelo índice de Jonhston.

\begin{tabular}{|l|c|c|c|c|c|}
\hline Grupos musculares & Ex.1 & Ex.2 & Ex.3 & Ex.4 & Ex.5 \\
\hline flexores de quadril & 2 & 2 & 2 & 2 & 2 \\
\hline extensores de quadril & 2 & 2 & 2 & 2 & 2 \\
\hline adutores de quadril & 4 & 4 & 4 & 4 & 4 \\
\hline abdutores de quadril & 2 & 2 & 2 & 2 & 2 \\
\hline rotadores medial de quadril & 4 & 4 & 4 & 4 & 4 \\
\hline rotadores lateral de quadril & 2 & 2 & 2 & 2 & 2 \\
\hline flexores de joelho & 2 & 2 & 2 & 2 & 2 \\
\hline extensores de joelho & 3 & 3 & 3 & 3 & 3 \\
\hline flexores de tornozelo & 2 & 2 & 2 & 2 & 2 \\
\hline extensores de tornozelo & 4 & 4 & 4 & 4 & 4 \\
\hline Ex. = Examinador & & & & \\
\hline
\end{tabular}

Quadro 2 - Representação dos resultados da avaliação do tono muscular dos membros inferiores realizada pelos 5 examinadores criança diparética

\begin{tabular}{|l|c|c|c|c|c|}
\hline Atividade & Ex.1 & Ex.2 & Ex.3 & Ex.4 & Ex.5 \\
\hline engatinhar & 2 & 2 & 2 & 2 & 2 \\
\hline marcha & 1 & 1 & 1 & 1 & 1 \\
\hline Ex. = Examinador
\end{tabular}

Quadro 4 - Apresenta os dados referentes a avaliação das atividades funcionais dinâmicas realizada pelos cinco examinadores, numa das crianças. 


\section{DISCUSSÃO}

Este método de avaliação busca avaliar a evolução do desempenho motor e funcional de crianças com PC submetidas à Fisioterapia, utilizando para tanto a mensuração objetiva das mudanças ocorridas no tono muscular e nas atividades funcionais com base nas características do desempenho motor de acordo com os princípios que regem o seu controle neural.

Nesse estudo, comprovou-se que essa escala do tono muscular - inicialmente desenvolvida em adultos — também é aplicável em crianças, com 100\% de concordância (Quadros 1 e 2). Consideramo-la também coerente, baseado na análise da distribuição das alterações tônicas dos sujeitos avaliados, pela qual observamos que todos os sujeitos mostraram alteração de tono mais significativa nos músculos extensores fisiológicos (antigravitários) (Quadros 1 e 2). É interessante ressaltar que consideramos uma alteração de tono mais significativa quando há uma menor capacidade de adaptação do fuso muscular ao movimento, manifestada pela presença de uma reação ao alongamento variável com a velocidade e presente durante uma parte do arco de movimento ou durante todo o arco de movimento.

A utilização da capacidade de adaptação do fuso muscular como parâmetro de avaliação das alterações de tono nos assegura a validade de construção e de conteúdo dessa escala.

As vantagens desse método de avaliação do tono muscular são inúmeras: objetividade da graduação; utilização de parâmetros neurofisiológicos na classificação; baixo custo (ausência de uso de equipamentos auxiliares); possibilidade de avaliação na ausência de motricidade voluntária e facilidade de aplicação.

A escala utilizada para avaliação da funcionalidade foi baseada no modelo utilizado em crianças com outras patologias neurológicas ${ }^{(10)}$. Seu propósito é avaliar quantitativa e qualitativamente as atividades funcionais.

Nesse estudo, comprovou-se que a escala - após as adaptações previstas em um estudo piloto ${ }^{(9)}$ — foi aplicável também em crianças com PC. Em virtude da escala ter sido desenvolvida ${ }^{(10)}$, adaptada e testada previamente ${ }^{(9)}$ e neste estudo ter sido replicada com 100\% de concordância (Quadros 3 e 4), consideramo-la também fidedigna.
Essa escala utiliza como parâmetro de avaliação as aquisições motoras e o controle postural nas diversas atividades funcionais da seqüência do desenvolvimento neuromotor. Assim, asseguramos a validade de construção e de conteúdo dessa escala.

Outras vantagens dessa escala são: possibilidade de utilização em crianças de qualquer idade, já que não prevê um escore ideal para a idade, apenas se há aumento significativo do escore com o passar do tempo; a utilização de itens avaliados rotineiramente pelos fisioterapeutas, facilitando sua aplicação correta; ênfase na independência funcional; ênfase no controle das aquisições motoras grosseiras.

Ainda que essa escala não avalie diretamente a motricidade fina, acreditamos que qualquer evolução visível nesta motricidade seja precedida por um incremento no controle proximal. Assim, o controle proximal é mais sensível a mudanças do que o controle fino de movimento.

O melhor atributo desse método é ter sido fundamentado através de uma indiscutível estruturação teórica que envolve a fisiologia do fuso muscular ${ }^{(5,19,24)}$ e do controle postural ${ }^{(15,26)}$. A graduação das escalas de tono muscular e funcionalidade representam pontos específicos da fisiopatologia envolvida na alteração tônica e/ou funcional. Por outro lado, sua aplicação é simples e provê informações importantes tanto em clínica quanto em pesquisa.

\section{CONCLUSÃO}

Este estudo confirmou a aplicabilidade e também fidedignidade de um protocolo de avaliação do tono muscular desenvolvido para adultos com disfunções neurológicas e para crianças com Paralisia Cerebral, como também a aplicabilidade e fidedignidade de um protocolo de avaliação da funcionalidade em crianças com PC.

A aplicação destes protocolos de avaliação na rotina clínica permitiria de maneira rápida e com baixo custo identificar quais os componentes das funções ou habilidades motoras que estão por se desenvolver ou controlar e assim implementar a ação terapêutica 


\section{REFERÊNCIAS BIBLIOGRÁFICAS}

1. Barraquér-Bordas, L.; Ponces Vergé, J.; Corominas Vigneaux, J.; Torras de Bea, E.; Noguer Rodriguez, L. A. La parálisis cerebral infantil. Barcelona: Editorial Científico-Médica, 1966 apud Schwartzman, J. S. Paralisia Cerebral. Temas sobre desenvo/vimento, v. 3, n. 13, p. 4-21, 1993.

2. Bobath, K. Uma Base Neurofisiológica para o Tratamento da Paralisia Cerebral. 2.ed. São Paulo: Manole. 1990, 110p.

3. Bower, E.; McLellan, D. L. Evaluating Therapy in Cerebral Palsy. Child Care Health Dev., v. 20, n. 6, p. 409-19, 1994

4. Boyce, W. F.,; Gowland, C.; Hardy, S.; Rosenbaum, P. L.; Lane, M.; Plews, N.; Goldsmith, C.; Russel, D. Development of a Quality-ofMovement measure for Children with Cerebral Palsy. Phys. Ther., v. 71, n. 11, p. 820-32, 1991

5. Boyce, W. F.; Gowland, C.; Lane, M.; Plews, N.; Goldsmith, C.; Russel, D. J.; Wright, V.; Zdrobov, S. Measuring Quality of Movement in Cerebral Palsy: A review of Instruments. Phys. Ther., v. 71, n. 11, p. 8139, 1991.

6. Boyce, W. F.; Gowland, C.; Rosenbaum, P. L.; Lane, M.; Plews, N.; Goldsmith, C.; Russel, D. J.; Wright, V.; Poter, S; Harding, D. The Gross Motor Performance Measure: validity and Responsiveness of a Measure of Quality of Movement. Phys. Ther., v. 75, n. 7, p. 603-13, 1995.

7. Brunnström, S. Motor Testing Procedures in Hemiplegia. J. Am. Phys. Ther. Ass., v. 46, p.357, 1966

8. Durigon, O. F. S.; Piemonte, M. E. P. Desenvolvimento de Protocolo para Avaliação do Tono Muscular. In: Congresso Brasileiro de Fisioterapia, 9. e Congresso Paulista de Fisioterapia, 4., 1993, São Paulo. Anais... São Paulo: ABF, 1993. p. 31.

9. Durigon, O. F S : Sá C S C. Sita L. V Desenvolvimento de um Protocolo de Avaliação do Desempenho Motor e Funcional de Crianças com Paralisia Cerebral. Arquivos de Neuro-Psiquiatria. v. 54, paper 24, 1996. Suplemento II.

10/11. Durigon, O. F. S.; Storto, J. N. Intervenção Fisioterápica em Crianças portadoras de Síndrome de Rett. In: Reunião Anual da Sociedade Brasileira para o Progresso da Ciência - SBPC, 48., 1996, São Paulo. Anais. Durigon, O. F.S; Sá, C.S.C; Sita, L.V Evaluation of the muscular tonus and function in cerebral Palsied children. Anais do $13^{\text {th }}$ International Congress of the WCPT,PL-RR-262-26B. Yokohama - Japan, May. 23-28, 1999.

12. Engsberg, J. R.; Olree, K. S.; Ross, S. A.; Park, T. S. Quantitative Clinical Measure of Spasticity in Children with Cerebral Palsy. Arch. Phys. Med. Rehabil., v. 77, p. 594-9, 1996.

13. Frankenburg, W. K.; Sciarillo, W.; Burgess, D. The newly abbreviated and revised Denver Developmental Screening Test. J. Pediatr., v. 99, n. 6, p. 995-9, 1981

14. Fugl-Meyer, A. R.; Jääsko, L.; Leyman, I.; Olsson, S.; Steiglind, S. Scand. J. Rehab. Med., v. 7, p. 13-31, 1975.

15. Ghez, C. Posture. In: Kandell, E. R.; Schwartz, J. H.; Jessel, T. M. Principles of Neural Science. 3. ed. Connecticut: Appleton \& Lange, 1991. p. 596607.

16. Glascoe, F. P.; Byrne, K. E. The Usefulness of the Battelle Developmental Inventory Screening Test. Clin. Pediatr. (Phila.), v. 32, n. 5, p. 273-80, 1993.

17. Glascoe F. P.; Byrne, K. E.; Ashford, L. G.; Johnson, K. L.; Chang, B.; Strickland, B. Accuracy of Denver-II in Developmental Screening. Pedi- atrics, v. 89, n. 6, 1982.

18. Glascoe, F. P.; Martin, E. D.; Humphrey, S. A Comparative Review of Developmental Screening Tests. Pediatrics, v. 86, n. 4, p. 547-54, 1990.

19. Gordon, J; Ghez, C. Muscle Receptors and Spinal Reflexes: The Stretch Reflex. In: Kandell, E. R.; Schwartz, J. H.; Jessel, T. M. Principles of Neural Science. 3. ed. Connecticut: Appleton \& Lange, p. 564-80, 1991

20. Gowland, C.; Boyce, W. F.; Wright, V.; Russel, D. J.; Goldsmith, C. H.; Rosenbaum, P. L. Reliability of the Gross Motor Performance Measure. Phys. Ther., v. 75, n. 7, p. 597-602, 1995

21. Guyatt, G.; Walter, S.; Norman, G. Measuring Change Over Time: Assessing the Usefulness of Evaluative Instruments. J. Chron. Dis., v. 40, n. 2, p. 171-8, 1987

22. Harris, S. R.; Heriza, C. B. Measuring Infant Movement. Phys. Ther., v. 67, n. 12, p. $1877-1880,1987$.

23. Henneman, E. Funções motoras do tronco cerebral e dos gânglios da base do encéfalo. In: Mountcastle, V. B. Fisiologia Médica. 13. ed. Rio de Janeiro: Guanabara Koogan, v.1. p. 680-705, 1985

24. Henneman, E. Mecanismos periféricos envolvidos no controle do músculo. In: Mountcastle, V. B. Fisiologia Médica. 13. ed. Rio de Janeiro: Guanabara Koogan, 1985. v.1. p. 619-37

25. Kendall, F. P.; McCreary, E. K. Músculos: Provas e Funções. 3. Ed. São Paulo: Manole. 1987, $380 \mathrm{p}$.

26. Mello Aires, M. Fisiologia Básica. Rio de Janeiro: Guanabara Koogan, 1985. P. 208-14.

27.. Nickel, R. E.; Renken, C. A.; Gallenstein, J, S. The Infant Motor Screen. Dev. Med. Child Neurol., v. 31, p. 35-42, 1989

28. Palisano, R. J.; Kolobe, T. H.; Haley, S. M.; Lowes, L. P.; Jones, S. L. Validity of the Peabody Developmental Gross Motor Scale as an Evaluative Measure of Infants Receiving Physical Therapy. Phys. Ther., v. 75, n. 11, p. 939-51, 1995.

29. Piper, M. C.; Pinnell, L. E.; Darrah, J.; Maguire, T.; Byrne, P. Construction and Validation of the Alberta Infant Motor Scale (AIMS). Canadian Journal of Public Health, v.83, p. 46-50, 1992. Supplement.

30. Rosenbaum, P. L.: Russel, D. J.; Cadman, D. T: Gowland, C.: Jarvis, S.: Hardy, S. Issues in Measuring Change in Motor Function In Children with Cerebral Palsy: A Special Communication. Phys. Ther., v. 70, n. 2, p. 125-131, 1990.

31. Rothstein, J. M.; Echternach, J. L. Primer on Measurement: An Introductory Guide to Measurement Issues. American Physical Therapy Association, 1993.

32/33. Russel, D. J.; Rosenbaum, P. L.; Cadman, D. T.; Gowland, C.; Hardy, S.; Jarvis, S. The Gross Motor Function Measure: A Means to Evaluate the Effects of Physical Therapy. Dev. Med. Child Neurol., v. 31, p. 341-52, 1989.ShumwayCook, A.; Woollacott, M. Motor control - Theory and pratical applications, Ed. Williams e Wilkins, 2000

34/35. Simeonsson, R. J.; Cooper, D. H.; Scheiner, A. P. A Review and Analysis of Early Intervention Programs. Pediatrics, v. 69, n. 5, p. 635-41, 1982.Palisano, R.J.; Hanna, S.e.; Rosenbaumm: Russell, D.J.; Walter, S.D.; Wood.E.P. Raima.P.S.: Galuppi. B.E. Validation of a model of Gross motor Function for children with CP, Physical Therapy, V.80, n.10, p.974-985, oct.2001 